\title{
A Special Permutation Matrix Decomposition for Combinatorial Design Incidence Matrices*
}

\author{
E. C. Johnsen** and T. F. Storer***
}

(March 9, 1971)

\begin{abstract}
By a well-known theorem of König, every 0,1 matrix $A$ of order $v$ with all row and column sums equal to $k>0$ can be decomposed into a sum of $k$ permutation matrices of order $v$. Here we consider whether $A$, trace $A=0$ or $v$, can have a special permutation matrix decomposition called a special König decomposition. The question is answered more or less satisfactorily using certain graph factorization theorems of Petersen and Tutte. Symmetric counterexamples are given to show that not all such matrices have special König decompositions; however, it is shown that symmetric incidence matrices for $\langle v, k, \lambda\rangle$ designs always have special König decompositions.
\end{abstract}

Key words: Block design; combinatorial analysis; configurations; König's theorem; matrices; 0,1 matrices; matrix equations; permutation matrix decompositions.

\section{Introduction}

$A\langle v, k\rangle$ system $[S, \mathscr{S}]$ is a set of $v$ distinct elements $S=\left\{x_{1}, x_{2}, \ldots, x_{v}\right\}$ together with a selection of $v$ not necessarily distinct subsets of $S, \mathscr{S}=\left\{X_{1}, X_{2}, \ldots, X_{v}\right\}$ such that each $X_{j}$ contains exactly $k$ of the elements in $S$ and each $x_{i}$ appears in exactly $k$ of the subsets in $S, 0 \leqslant k \leqslant v$. If, in addition, we have $\left|X_{1} \cap X_{j}\right|=\lambda$ for each $X_{j}$, where $0<\lambda<k<v-1$, then we call [S, $\left.\mathscr{S}\right]$ a $\langle v, k, \lambda\rangle$ principal block partial design, where $X_{1}$ is the principal block. If, further, we have $\left|X_{i} \cap X_{j}\right|=\lambda$ for every pair $X_{i}, X_{j}, i \neq j$, where $0<\lambda<k<v-1$, then $[S, \mathscr{S}]$ is known as a $\langle v, k, \lambda\rangle$ design. When $[S, \mathscr{S}]$ is a $\langle v, k, \lambda\rangle$ principal block partial design then, by a simple counting argument, we have:

$$
(v-1) \lambda=k(k-1) .
$$

The incidence matrix of a $\langle v, k\rangle$ system $[S, \mathscr{S}]$ is the 0,1 matrix $A=\left[a_{i j}\right]$ of order $v$ where:

$$
a_{i j}= \begin{cases}1, & x_{j} \in S_{i} \\ 0, & x_{j} \notin S_{i}\end{cases}
$$

Note that each row and column sum of $A$ is $k$. We denote the set of all such 0,1 matrices by $\mathfrak{A}_{v}(k)$. Without loss of generality, we assume that the elements in $S$ and the sets in $\mathscr{S}$ are ordered so that either $x_{i} \notin X_{i}$ for all $i=1,2, \ldots, v$, or $x_{i} \in X_{i}$ for all $i=1,2, \ldots, v$. Then we have either $\operatorname{tr} A=0$ or $\operatorname{tr} A=v$, respectively. Now, by a well-known theorem of König [3; p. 239, B.], $A$ can be written as the sum of $k$ permutation matrices of order $v$ :

$$
A=\delta I+\sum_{s=1}^{k-\delta} P_{r_{s}}, \quad \delta=\left\{\begin{array}{ll}
0, & \operatorname{tr} A=0 \\
1, & \operatorname{tr} A=v
\end{array},\right.
$$

AMS Subject Classification: Primary 0520, Secondary 0525, 0530, 1535

*An invited paper. Supported in part by Air Force Office of Scientific Research Grant AFOSR 698-67 and in part by an NSF Research Grant.

**Present address: The University of California, Santa Barbara, Calif. 93106.

***Present address: The University of Michigan, Ann Arbor, Mich. 48104. 
where $P_{r_{s}}$ is the permutation matrix whose 1 in row 1 is in column $r_{s}$. We call (1.3) a König decomposition of $A$. If $B=\left[b_{i j}\right]$ and $c=\left[c_{i j}\right]$ are two 0,1 matrices of order $v$, we write $B \cap C \equiv E$ for the matrix $E=\left[e_{i j}\right]$ of order $v$ where $e_{i j}=\min \left\{b_{i j}, c_{i j}\right\}, i, j=1,2, \ldots, v$. We say that $A$ contains the permutation matrix $P$ or that $P$ is contained in $A$ if $A \cap P=P$.

In recent investigations on the above combinatorial designs [2] the authors encountered the following rather interesting problem. Let $A$ be the incidence matrix of a $\langle v, k\rangle$ system. When is it possible to find a König decomposition (1.3) of $A$ such that for each $P_{r_{s}}$ in the decomposition either $P_{r_{s}}^{T} \cap A=0$ or $P_{r_{s}}^{T} \cap A=P_{r_{t}}$ for some $P_{r_{t}}$ in the decomposition? We call such a decomposition a special König decomposition of $A$. (If $A=0$ or $A=I$ then $A$ is considered to vacuously have a special König decomposition.) When $P_{r_{s}}^{T} \cap A=0, P_{r_{s}}=\left[p_{i j}\right]$ has the properties that $\operatorname{tr} P_{r_{s}}=0$ and for no $i, j, i \neq j$, does $p_{i j}=p_{j i}=1$. We call such a permutation matrix skew. When $P_{r_{s}}^{T} \cap A=P_{r_{t}}$ for some $P_{r_{t}}$ then $P_{r_{t}}=P_{r_{s}}^{T}$ and either $P_{r_{s}}$ is skew or else $P_{r_{s}}^{T}=P_{r_{t}}=P_{r_{s}}$ and $P_{r_{s}}$ is a symmetric permutation matrix with $\operatorname{tr} P_{r_{s}}=0$. In the latter case we call $P_{r_{s}}$ a 0 -symmetric permutation matrix. Thus, a special König decomposition of $A$ has the form

$$
A=\delta I+\sum_{\alpha} P_{r_{\alpha}}+\sum_{\beta}\left(P_{r_{\beta}}+P_{r_{\beta}}^{T}\right)+\sum_{\gamma} P_{r_{\gamma}}
$$

where the $P_{r_{\alpha}}$ 's are the skew permutation matrices whose transposes do not occur in the decomposition, the $P_{r_{\beta}}$ 's are the skew permutation matrices whose transposes do occur in the decomposition, and the $P_{r \gamma}$ 's are the 0 -symmetric permutation matrices in the decomposition.

The following brief remarks are intended to convey the interest in the special König decomposition. In [2] the authors show that every abstract $\langle v, k\rangle$ system $[S, \mathscr{S}]$ can be isomorphically identified with a $\langle v, k\rangle$ system in an additive loop $\mathscr{L}$ of order $v$. In this identification, the elements of $[S, \mathscr{S}]$ correspond to the elements of $\mathscr{L}$ and the sets of $[S, \mathscr{S}]$ correspond to the left translates $l+D, l \epsilon \mathscr{L}$, of a $k$-subset $D$ of $\mathscr{L}$. The identification is obtained via König decompositions of the incidence matrices $A$ of $[S, \mathscr{S}]$ and $A_{c}$ of the complementary system $\left[S, \mathscr{S}_{c}\right], \mathscr{S}_{c}=\left\{\mathscr{S}-X_{1}, \ldots, S-X_{v}\right\}$. Under this identification, the set of elements $D$ is right inversive in $\mathscr{L}$ if and only if $A$ has a special König decomposition, and $\mathscr{L}$ is a right inverse property loop if and only if both $A$ and $A_{c}$ have special König decompositions. When $D$ is right inversive, $D$ forms a difference set in $\mathscr{L}$ if and only if $[S, \mathscr{S}]$ is a $\langle v, k, \lambda\rangle$ principal block partial design. This situation has further combinatorial implications, which can be found in [2].

Since not all $\langle v, k\rangle$ system incidence matrices $A$, with $\operatorname{tr} A=0$ or $\operatorname{tr} A=v$, have a special König decomposition, we need to find conditions on $A$ such that $A$ does have such a decomposition. Let us suppose that $A$ has a special König decomposition. We consider a pair $a_{i j}, a_{j i}, i \neq j$, where $a_{i j}=a_{j i}=1$. This pair of 1's must either be entries in a 0 -symmetric permutation matrix $P_{r_{\gamma}}$ or of a transpose-pair of skew permutation matrices $P_{r_{\beta}}, P_{r_{\beta}}^{T}$. All such pairs of 1's appear in the matrix $A_{s} \equiv A \cap A^{T}$. Note that $A_{q} \equiv A-A_{s}=\left[a_{i j}^{(q)}\right]$ has the property that if $a_{i j}^{(q)}=1, i \neq j$, then $a_{j i}^{(q)}=0$. Thus, a necessary condition for $A$ to have a special König decomposition is that $A_{s} \epsilon \mathfrak{U}_{v}\left(k_{s}\right)$ (whence $A_{q} \epsilon \mathfrak{U}_{v}\left(k-k_{s}\right)$ ) for some $k_{s}, 0 \leqslant k_{s} \leqslant k$. Now, a special König decomposition of $A$ induces special König decompositions of $A_{s}$ and $A_{q}$, and conversely. Since $A \cap A_{q}^{T}=0$, it is easy to see that every König decomposition of $A_{q}$ is a special König decomposition. Thus, we can reduce the problem to symmetric matrices:

Lemma 1.1: Let $\mathrm{A} \epsilon \mathfrak{U}_{\mathrm{v}}(\mathrm{k})$, where $\operatorname{tr} \mathrm{A}=0$ or $\operatorname{tr} \mathrm{A}=\mathrm{v}$. Then necessary and sufficient conditions for A to have a special König decomposition are that

(a) $\mathrm{A}_{\mathrm{s}} \in \mathfrak{U}_{\mathrm{v}}\left(\mathrm{k}_{\mathrm{s}}\right)$ for some $\mathrm{k}_{\mathrm{s}}, 0 \leqslant \mathrm{k}_{\mathrm{s}} \leqslant \mathrm{k}$, and

(b) $\mathrm{A}_{\mathrm{s}}$ have a special König decomposition.

For any given $A$, condition (a) is easy to decide. So we invoke this reduction and assume that $A$ is symmetric, i.e., $A=A_{s}, k=k_{s}$, and $A_{q}=0$. Here (1.4) becomes:

$$
A=\delta I+\sum_{\beta}\left(P_{r_{\beta}}+P_{r_{\beta}}^{T}\right)+\sum_{\gamma} P_{r_{\gamma}}
$$


We shall call $A$-symmetric or co-symmetric according as $\operatorname{tr} A=0$ or $\operatorname{tr} A=v$. We note that if $A$ contains a 0 -symmetric permutation matrix then $v$ must be even.

In this paper we settle more or less satisfactorily the question of whether a 0 -symmetric or co-symmetric $A$ has a special König decomposition by first transforming the question into a natural problem on regular graphs and then applying two theorems in graph theory concerning the existence of 1 -factors and 2-factors in such graphs. For the cases where only 2 -factors need be considered, the matrix $A$ always has a special König decomposition by a theorem of Petersen. For the cases where 1 -factors must be considered, the question is not as satisfactorily answered since the answer is determined by the criterion in Tutte's theorem on the existence of 1 -factors in a graph, and it is not easy, in general, to apply this criterion. In this regard, we show that in such cases $A$ need not have a special König decomposition. In fact, we exhibit 0 -symmetric and co-symmetric $A$ 's which are incidence matrices for $\langle v, k, \lambda\rangle$ principal block partial designs which do not have special König decompositions. Finally, however, we do show, using a corollary of Tutte's theorem for regular graphs, that in all cases where $A$ is the incidence matrix for a $\langle v, k, \lambda\rangle \operatorname{design}, A$ always has a special König decomposition.

\section{Main Results}

Again, let $A$ be a 0 -symmetric or co-symmetric 0,1 matrix in $\mathfrak{A}_{v}(k), 0 \leqslant k \leqslant v$. We define

$$
\hat{A}=\left\{\begin{array}{l}
A, \operatorname{tr} A=0 \\
A-I, \operatorname{tr} A=v .
\end{array}\right.
$$

Then $\hat{A}$ will be interpreted as the adjacency matrix of a regular graph $\mathscr{G}(V, \hat{k})$ of degree $\hat{k}$ on $v$ vertices $\boldsymbol{V}=\left\{v_{1}, \ldots, v_{v}\right\}$, in this order, where

$$
\hat{k}=\left\{\begin{array}{l}
k, \operatorname{tr} A=0 \\
k-1, \operatorname{tr} A=v .
\end{array}\right.
$$

An $m$-factor of $\mathscr{G}(V, \hat{k})$ is a subgraph $\mathscr{G}(V, m)$ of degree $m>0$ on the full set of vertices $V$, and we say that $\mathscr{G}(V, \hat{k})$ is factorable into regular factors $\mathscr{G}\left(V, m_{i}\right), i=1,2, \ldots, t$, if

$$
\mathscr{G}(V, \hat{k})=\sum_{i=1}^{t} \mathscr{G}\left(V, m_{i}\right)
$$

where the summation indicates that the set of edges in $\mathscr{G}(V, \hat{k})$ is the union of the sets of edges in the factors $\mathscr{G}\left(V, m_{i}\right)$. (For $t=0$ this is the vacuous factorization for either $A=0$ or $A=I$.) We note that the adjacency matrix of a l-factor of $\mathscr{G}(V, \hat{k})$ is a 0 -symmetric permutation matrix contained in $A$, and that the adjacency matrix of a 2 -factor of $\mathscr{G}(V, \hat{k})$ is the sum of a skew permutation matrix and its transpose, both of which are contained in $A$. Hence, by (1.5) we have:

LEMMA 2.1: A has a special König decomposition if and only if $\mathscr{G}(V, \hat{k})$ is factorable into regular factors of degrees 1 and 2.

We first note that when $v$ is odd and $\hat{k}$ is odd Lemma 2.1 requires that $\mathscr{G}(V, \hat{k})$ have a 1 -factor, which means that $v$ must be even, a contradiction. Actually, $A$ does not even exist in this case, since here the total sum of vertex degrees in $\mathscr{G}(V, \hat{k})$ would be $v \hat{k}$, which is odd, but would also be twice the number of edges in $\mathscr{G}(V, \hat{k})$, which is even, a contradiction. Thus,

THEOREM 2.2: A does not exist for $\mathrm{v}$ odd when

(i) $k$ is odd and $\operatorname{tr} \mathrm{A}=0$.

(ii) $\mathrm{k}$ is even and $\operatorname{tr} \mathrm{A}=v$. 
Now, relevant to our investigation here for other cases is the following result due to Petersen [4]:

THEOREM 2.3. A regular graph of even degree $\mathrm{d}=2 \mathrm{e}$ is factorable into e 2 -factors.

By this theorem we then have

THEOREM 2.4: A has a special König decomposition when

(i) $\mathrm{k}$ is even and $\operatorname{tr} \mathrm{A}=0$.

(ii) $\mathrm{k}$ is odd and $\operatorname{tr} \mathrm{A}=\mathrm{v}$.

By Theorems 2.2 and 2.4 the remaining undecided cases are for $v$ even with either $k$ odd and $\operatorname{tr} A=0$ or $k$ even and $\operatorname{tr} A=\boldsymbol{v}$. In these cases the graph $\mathscr{G}(V, \hat{k})$ has $\hat{k}$ odd. Thus, if $A$ is to have a special König decomposition here, $\mathscr{G}(V, \hat{k})$ must have a 1 -factor. Further, this condition is sufficient since then the subgraph of $\mathscr{G}(V, \hat{k})$ remaining after removal of a 1 -factor would be regular of even degree $\hat{k}-1$, which, by Theorem 2.3 , would factor into $(\hat{k}-1) / 22$-factors. Relevant to our investigation here is the following result due to Tutte [5]:

THEOREM 2.5: Let $\mathscr{G}$ be a connected graph on a set of vertices $\mathrm{X}$. For any proper subset $\mathrm{Y}$ of $\mathrm{X}$ let $\theta(\mathrm{Y})$ denote the number of components of odd order in the subgraph of $\mathscr{G}$ determined by the vertices $\mathrm{X}-\mathrm{Y}$. Then, a necessary and sufficient condition for $\mathscr{G}$ to have a 1 -factor is that $\theta(\mathrm{Y}) \leqslant|\mathrm{Y}|$ for every proper subset $\mathrm{Y}$ of $\mathrm{X}$.

Applying this theorem to the 0 -symmetric or co-symmetric matrix $A$ we have:

THEOREM 2.6: A has a special König decomposition when

(i) $\mathrm{k}$ is odd and $\operatorname{tr} \mathrm{A}=0$, or

(ii) $\mathrm{k}$ is even and $\operatorname{tr} \mathrm{A}=\mathrm{v}$,

if and only if for every irreducible direct summand $\mathrm{A}^{\prime}$ of $\mathrm{A}, \mathrm{A}^{\prime}$ is of even order and the necessary and sufficient condition of Theorem 2.5 holds for the component of $\mathscr{G}(\mathrm{V}, \hat{\mathrm{k}})$ whose adjacency matrix is $\mathrm{A}^{\prime}$.

We apply Theorem 2.5 to show that in these two undecided cases $A$ need not have a special König decomposition. In fact, our counterexamples will be 0 -symmetric and co-symmetric incidence matrices for $\langle v, k, \lambda\rangle$ principal block partial designs.

Counterexample 1. $v$ even, $k$ odd, $\operatorname{tr} A=0: v=106, k=15, \lambda=2$.

Let $\bar{e}=\left[\begin{array}{lllll}1 & 1 & 1 & 1 & 1\end{array}\right], \overline{0}=\left[\begin{array}{llllll}0 & 0 & 0 & 0 & 0 & 0\end{array}\right]$, and form $\bar{u}=[\bar{e}, \overline{0}, \overline{0}, \overline{0}, \overline{0}, \overline{0}]$ of length 35 .

Let

$B=\left[\begin{array}{lllll}0 & 1 & 1 & 0 & 0 \\ 1 & 0 & 0 & 1 & 0 \\ 1 & 0 & 0 & 0 & 1 \\ 0 & 1 & 0 & 0 & 1 \\ 0 & 0 & 1 & 1 & 0\end{array}\right]$,

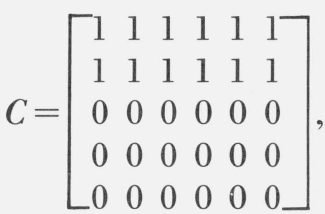

$D=\left[\begin{array}{llllll}1 & 1 & 1 & 1 & 1 & 1 \\ 0 & 0 & 0 & 0 & 0 & 0 \\ 1 & 1 & 1 & 1 & 1 & 1 \\ 0 & 0 & 0 & 0 & 0 & 0 \\ 0 & 0 & 0 & 0 & 0 & 0\end{array}\right]$,

$\boldsymbol{E}=\left[\begin{array}{llllll}0 & 0 & 0 & 0 & 0 & 0 \\ 1 & 1 & 1 & 1 & 1 & 1 \\ 0 & 0 & 0 & 0 & 0 & 0 \\ 1 & 1 & 1 & 1 & 1 & 1 \\ 0 & 0 & 0 & 0 & 0 & 0\end{array}\right]$,

$F=\left[\begin{array}{llllll}0 & 0 & 0 & 0 & 0 & 0 \\ 0 & 0 & 0 & 0 & 0 & 0 \\ 1 & 1 & 1 & 1 & 1 & 1 \\ 0 & 0 & 0 & 0 & 0 & 0 \\ 1 & 1 & 1 & 1 & 1 & 1\end{array}\right]$,

$$
G=\left[\begin{array}{llllll}
0 & 0 & 0 & 0 & 0 & 0 \\
0 & 0 & 0 & 0 & 0 & 0 \\
0 & 0 & 0 & 0 & 0 & 0 \\
1 & 1 & 1 & 1 & 1 & 1 \\
1 & 1 & 1 & 1 & 1 & 1
\end{array}\right],
$$

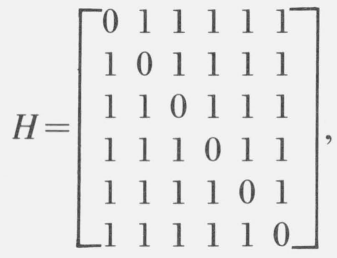

$K=\left[\begin{array}{llllll}1 & 1 & 1 & 1 & 1 & 1 \\ 0 & 0 & 0 & 0 & 0 & 0 \\ 0 & 0 & 0 & 0 & 0 & 0 \\ 1 & 1 & 1 & 1 & 1 & 1 \\ 0 & 0 & 0 & 0 & 0 & 0 \\ 0 & 0 & 0 & 0 & 0 & 0\end{array}\right]$, 
$L=\left[\begin{array}{llllll}1 & 1 & 0 & 0 & 0 & 0 \\ 0 & 0 & 1 & 1 & 1 & 1 \\ 0 & 0 & 0 & 0 & 0 & 0 \\ 1 & 1 & 0 & 0 & 0 & 0 \\ 0 & 0 & 1 & 1 & 1 & 1 \\ 0 & 0 & 0 & 0 & 0 & 0\end{array}\right], \quad M=\left[\begin{array}{llllll}0 & 0 & 0 & 0 & 0 & 0 \\ 1 & 1 & 1 & 1 & 0 & 0 \\ 0 & 0 & 0 & 0 & 1 & 1 \\ 0 & 0 & 0 & 0 & 0 & 0 \\ 1 & 1 & 1 & 1 & 0 & 0 \\ 0 & 0 & 0 & 0 & 1 & 1\end{array}\right], \quad N=\left[\begin{array}{llllll}0 & 0 & 0 & 0 & 0 & 0 \\ 0 & 0 & 0 & 0 & 0 & 0 \\ 1 & 1 & 1 & 1 & 1 & 1 \\ 0 & 0 & 0 & 0 & 0 & 0 \\ 0 & 0 & 0 & 0 & 0 & 0 \\ 1 & 1 & 1 & 1 & 1 & 1\end{array}\right], \quad Q=\left[\begin{array}{llllll}0 & 0 & 0 & 0 & 0 & 0 \\ 1 & 1 & 1 & 1 & 1 & 1 \\ 0 & 0 & 0 & 0 & 0 & 0 \\ 0 & 0 & 0 & 0 & 0 & 0 \\ 1 & 1 & 1 & 1 & 1 & 1 \\ 0 & 0 & 0 & 0 & 0 & 0\end{array}\right]$,

$R=\left[\begin{array}{llllll}1 & 1 & 1 & 1 & 0 & 0 \\ 0 & 0 & 0 & 0 & 1 & 1 \\ 0 & 0 & 0 & 0 & 0 & 0 \\ 1 & 1 & 1 & 1 & 0 & 0 \\ 0 & 0 & 0 & 0 & 1 & 1 \\ 0 & 0 & 0 & 0 & 0 & 0\end{array}\right], \quad S=\left[\begin{array}{llllll}0 & 0 & 0 & 0 & 0 & 0 \\ 1 & 1 & 0 & 0 & 0 & 0 \\ 0 & 0 & 1 & 1 & 1 & 1 \\ 0 & 0 & 0 & 0 & 0 & 0 \\ 1 & 1 & 0 & 0 & 0 & 0 \\ 0 & 0 & 1 & 1 & 1 & 1\end{array}\right], \quad T=\left[\begin{array}{llllll}1 & 1 & 0 & 0 & 0 & 0 \\ 0 & 0 & 1 & 1 & 0 & 0 \\ 0 & 0 & 0 & 0 & 1 & 1 \\ 1 & 1 & 0 & 0 & 0 & 0 \\ 0 & 0 & 1 & 1 & 0 & 0 \\ 0 & 0 & 0 & 0 & 1 & 1\end{array}\right]$,

and form

$$
U=\left[\begin{array}{llllll}
B & C & D & E & F & G \\
C^{T} & H & K & L & M & N \\
D^{T} & K^{T} & H & K & Q & N \\
E^{T} & L^{T} & K^{T} & H & R & S \\
F^{T} & M^{T} & Q^{T} & R^{T} & H & T \\
G^{T} & N^{T} & N^{T} & S^{T} & T^{T} & H
\end{array}\right]
$$

of order 35. Let $\mathbf{0}$ denote the zero matrix of order 35. Finally, form

$$
\mathrm{A}_{106}=\left[\begin{array}{llll}
0 & \bar{u} & \bar{u} & \bar{u} \\
\bar{u}^{T} & U & \mathbf{0} & \mathbf{0} \\
\bar{u}^{T} & \mathbf{0} & U & \mathbf{0} \\
\bar{u}^{T} & \mathbf{0} & \mathbf{0} & U
\end{array}\right]
$$

of order 106 with row sums and column sums all equal to 15 and all inner products of row 1 with any other row equal to 2 . Let $\mathscr{G}_{106}$ be the connected graph of degree 15 on the 106 vertices $V=\left\{v_{1}, v_{2}, \ldots, v_{106}\right\}$ whose adjacency matrix is $A_{106}$. For $\mathrm{Y}=\left\{v_{1}\right\}, \theta(Y)=3>1=|Y|$, hence, by Theorem $2.5, \mathscr{G}_{106}$ does not have a 1 -factor, whence $A_{106}$ does not have a special König decomposition.

Counterexample 2. $v$ even, $k$ even, $\operatorname{tr} A=v: v=46, k=10, \lambda=2$.

Let $\bar{e}=\left[\begin{array}{lll}1 & 1 & 1\end{array}\right], \overline{0}=\left[\begin{array}{llll}0 & 0 & 0 & 0\end{array}\right]$ and form $\bar{w}=[\bar{e}, \overline{0}, \overline{0}, \overline{0}]$ of length 15 .

Let

$$
\begin{aligned}
& I_{3}=\left[\begin{array}{lll}
1 & 0 & 0 \\
0 & 1 & 0 \\
0 & 0 & 1
\end{array}\right], B=\left[\begin{array}{llll}
1 & 1 & 1 & 1 \\
1 & 1 & 1 & 1 \\
0 & 0 & 0 & 0
\end{array}\right], C=\left[\begin{array}{llll}
1 & 1 & 1 & 1 \\
0 & 0 & 0 & 0 \\
1 & 1 & 1 & 1
\end{array}\right], D=\left[\begin{array}{llll}
0 & 0 & 0 & 0 \\
1 & 1 & 1 & 1 \\
1 & 1 & 1 & 1
\end{array}\right], \\
& J_{4}=\left[\begin{array}{llll}
1 & 1 & 1 & 1 \\
1 & 1 & 1 & 1 \\
1 & 1 & 1 & 1 \\
1 & 1 & 1 & 1
\end{array}\right], E=\left[\begin{array}{llll}
1 & 1 & 1 & 1 \\
0 & 0 & 0 & 0 \\
1 & 1 & 1 & 1 \\
0 & 0 & 0 & 0
\end{array}\right], F=\left[\begin{array}{llll}
0 & 0 & 0 & 0 \\
1 & 1 & 1 & 1 \\
0 & 0 & 0 & 0 \\
1 & 1 & 1 & 1
\end{array}\right], G=\left[\begin{array}{llll}
1 & 1 & 0 & 0 \\
0 & 0 & 1 & 1 \\
1 & 1 & 0 & 0 \\
0 & 0 & 1 & 1
\end{array}\right],
\end{aligned}
$$

and form

$$
W=\left[\begin{array}{llll}
I_{3} & B & C & D \\
B^{T} & J_{4} & E & F \\
C^{T} & E^{T} & J_{4} & G \\
D^{T} & F^{T} & G^{T} & J_{4}
\end{array}\right]
$$


of order 15. Let $\mathbf{0}$ denote the zero matrix of order 15. Finally, form

$$
A_{46}=\left[\begin{array}{llll}
1 & \bar{w} & \bar{w} & \bar{w} \\
\bar{w}^{T} & W & \mathbf{0} & \mathbf{0} \\
\bar{w}^{T} & \mathbf{0} & W & \mathbf{0} \\
\bar{w}^{T} & \mathbf{0} & \mathbf{0} & W
\end{array}\right]
$$

of order 46 with row sums and column sums all equal to 10 and all inner products of row 1 with any other row equal to 2 . Let $\mathscr{G}_{46}$ be the connected graph of degree 9 on the 46 vertices $V=\left\{v_{1}\right.$, $\left.v_{2}, \ldots, v_{46}\right\}$ whose adjacency matrix is $A_{46}-I$. For $Y=\left\{v_{1}\right\}, \theta(Y)=3>1=|Y|$, hence, by Theorem $2.5, \mathscr{G}_{46}$ does not have a 1 -factor, whence $A_{46}$ does not have a special König decomposition.

We now show that a 0 -symmetric or co-symmetric incidence matrix for a $\langle v, k, \lambda\rangle$ design does have a special König decomposition. Relevant to the proof of this result is the following corollary to Theorem 2.5 [1; p. 182, Theorem 6].

CoRollaRY 2.7. Let $\mathscr{G}$ be a connected regular graph of degree $\mathrm{h}$ on a set $\mathrm{X}$ of an even number of vertices. For any proper subset $\mathrm{Y}$ of $\mathrm{X}$ let $\eta(\mathrm{Y})$ denote the number of edges in 9 which connect a vertex in $\mathrm{Y}$ with a vertex in $\mathrm{X}-\mathrm{Y}$. Then a sufficient condition for $\mathscr{G}$ to have a 1-factor is that $\eta(\mathrm{Y}) \geqslant \mathrm{h}-1$ for every proper subset $\mathrm{Y}$ of $\mathrm{X}$.

We note that the sufficient condition in this corollary is not a necessary condition, as is easily seen in the following graph of degree 3 on 10 vertices:

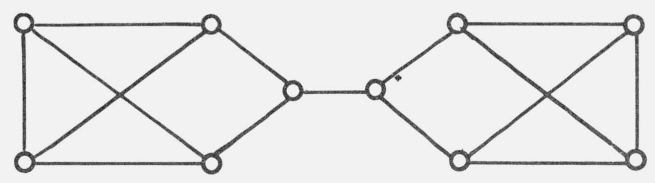

Now, the theorem for $\langle v, k, \lambda\rangle$ designs.

THEOREM 2.8. Let A be a 0 -symmetric or co-symmetric incidence matrix for $a\langle\mathrm{v}, \mathrm{k}, \lambda\rangle$ design. Then A has a special König decomposition.

Proof: By Theorems 2.2 and 2.4 we may limit our attention to the cases for $v$ even where either $k$ is odd and $\operatorname{tr} A=0$ or $k$ is even and $\operatorname{tr} A=v$. We choose any proper subset of $\mu$ rows of $A$ and we permute the rows of $A$ such that these $\mu$ rows become the first $\mu$ rows. We perform this same permutation on the columns of the resulting matrix. We then obtain a 0 -symmetric or cosymmetric matrix of the form

$$
P A P^{T}=\left[\begin{array}{ll}
F & E \\
E^{T} & G
\end{array}\right],
$$

where $P$ is the permutation matrix inducing the above permutation of the rows of $A, F$ is of order $\mu$, and $G$ is of order $v-\mu$. Let $e$ denote the number of 1's in $E$. Then the number of 1 's in $F$ is $\sigma=\mu k-e$, and the number of 1 's in $G$ is $\tau=(v-\mu) k-e$. Considering $\hat{A}$ as the adjacency matrix of a regular graph $\mathscr{G}(\boldsymbol{V}, \hat{k})$ and $Y$ as the set of $\mu$ vertices of $\mathscr{G}(V, \hat{k})$ given by the proper subset of $\mu$ rows and columns of $\hat{A}$, we see that $\eta(Y)=e$. Thus, since these $\mu$ vertices, $1 \leqslant \mu \leqslant v-1$, are arbitrary, if we can show that

$$
e \geqslant \hat{k}-1=\left\{\begin{array}{l}
k-1, \operatorname{tr} A=0 \\
k-2, \operatorname{tr} A=v
\end{array},\right.
$$

then, by Corollary 2.7 (since $e \geqslant k-2>0$ for any choice of $\mu$ vertices implies the graph is connected), we will have shown that $\mathscr{G}(V, \hat{k})$ has a 1 -factor, which, by our previous discussion, implies that $A$ has a special König decomposition. So, suppose that $e \leqslant k-2$. Then, since the number of entries in $F$ is $\mu^{2}$, we have

$$
\mu^{2} \geqslant \sigma=\mu k-e \geqslant \mu k-k+2,
$$


or

$$
\left(\mu-\frac{k}{2}\right)^{2} \geqslant\left(\frac{k}{2}\right)^{2}-k+2>\left(\frac{k}{2}-1\right)^{2},
$$

or, since $k>2$,

$$
\left|\mu-\frac{k}{2}\right|>\frac{k}{2}-1
$$

Now, if $\mu<\frac{k}{2}$ then (2.3) would imply that $\mu<1$, a contradiction. Hence, $\mu \geqslant \frac{k}{2}$ and from (2.3) we have

$$
\mu \geqslant k \text {. }
$$

By a similar argument, comparing the number of entries in $G$ with $\tau$, we obtain the corresponding result

$$
\mu \leqslant v-k \text {. }
$$

We now let $\sigma_{i}$ denote the sum of column $i$ in $F, 1 \leqslant i \leqslant \mu$. Then, counting all inner products of ordered pairs of rows of $F$, we see, noting that all entries are 0 or 1 , that

$$
\sum_{i=1}^{\mu} \sigma_{i}^{2} \leqslant \sigma+\mu(\mu-1) \lambda
$$

Now, by the theorem of the means,

$$
\frac{1}{\mu} \sum_{i=1}^{\mu} \sigma_{i}^{2} \geqslant\left(\frac{1}{\mu} \sum_{i=1}^{\mu} \sigma_{i}\right)^{2}=\frac{\sigma^{2}}{\mu^{2}},
$$

hence, by (2.6),

$$
\sigma^{2}-\mu \sigma \leqslant \mu^{2}(\mu-1) \lambda
$$

or

$$
\left(\sigma-\frac{\mu}{2}\right)^{2} \leqslant\left(\frac{\mu}{2}\right)^{2}\{4(\mu-1) \lambda+1\},
$$

whence, since $\sigma=\mu k-e$,

$$
\left|\mu\left(k-\frac{1}{2}\right)-e\right| \leqslant \frac{\mu}{2} \sqrt{4(\mu-1) \lambda+1} .
$$

Now, since $\mu \geqslant k>2$, if $e \geqslant \mu\left(k-\frac{1}{2}\right)$ then $e \geqslant \mu\left(k-\frac{1}{2}\right)>k-\frac{1}{2}>k-1$, which is contrary to our assumption that $e \leqslant k-2$. Hence $e<\mu\left(k-\frac{1}{2}\right)$ and (2.7) becomes

$$
e>\mu\left\{k-\frac{1}{2}-\frac{1}{2} \sqrt{4(\mu-1) \lambda+1}\right\} .
$$

Then, by (2.4), (2.5), and (2.8) we have

$$
e>k\left\{k-\frac{1}{2}-\frac{1}{2} \sqrt{4(v-k-1) \lambda+1}\right\}
$$


which, since $(v-1) \lambda=k^{2}-k$, becomes

$$
e>k\left\{k-\frac{1}{2}-\sqrt{\left(k-\frac{1}{2}\right)^{2}-k \lambda}\right\} .
$$

Now, since $v$ is even and $(v-1) \lambda=k(k-1)$ is always even, we have $\lambda$ even, implying $\lambda \geqslant 2$, whence $k(\lambda-2) \geqslant 0$, or

$$
k^{2}-3 k+\left(\frac{3}{2}\right)^{2} \geqslant k^{2}-k-k \lambda+\left(\frac{3}{2}\right)^{2},
$$

or

$$
\left(k-\frac{3}{2}\right)^{2} \geqslant\left(k-\frac{1}{2}\right)^{2}-k \lambda+2>\left(k-\frac{1}{2}\right)^{2}-k \lambda>0,
$$

whence, since $k>2$,

$$
k-\frac{3}{2}>\sqrt{\left(k-\frac{1}{2}\right)^{2}-k \lambda} .
$$

Combining (2.9) and (2.10) yields

$$
e>k\left\{k-\frac{1}{2}-k+\frac{3}{2}\right\}=k
$$

which is also contrary to our assumption that $e \leqslant k-2$. Hence, $e \geqslant k-1$ and by (2.2) we have the theorem.

\section{References}

[1] Berge, Claude, The Theory of Graphs and its Applications (translated by Alison Doig, Methuen and Co. Ltd., London, 1966).

[2] Johnsen, E. C., and T. F. Storer, Combinatorial structures in loops, I. Decomposition theory, submitted for publication.

[3] König, Dénes, Theorie der endlichen und unendlichen Graphen (Chelsea Publishing Company, New York, N.Y., 1950).
[4] Petersen, Julius, Die Theorie der regulären Graphs, Acta Math. 15, 193-220 (1891).

[5] Tutte, W. T., The factorization of linear graphs, J. London Math. Soc. 22, 107-111 (1947).

(Paper 75B1\&2-341) 